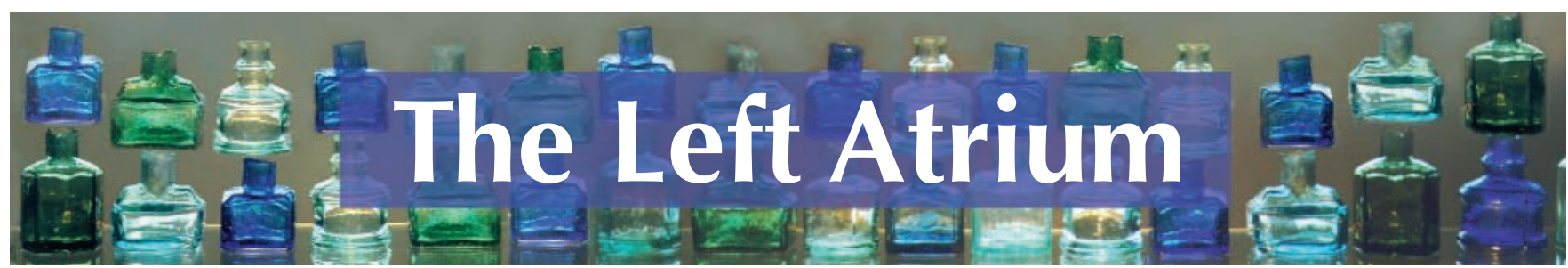

\section{Ethical investigations}

Double standards in medical research in developing countries

Ruth Macklin

Cambridge (UK): Cambridge University Press; 2004

288 pp $\$ 75$ (cloth) ISBN 0-521-83388-4

$\$ 34.99$ (paper) 0-521-54170-0

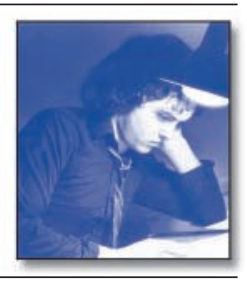

$\mathrm{R}$ uth Macklin, professor of bioethics at Albert Einstein College of Medicine in New York, has been publishing for many years on the theme of human rights protections in medical research and has held a number of prominent advisory positions with US and international organizations. In this, her most recent book, Macklin explores some of the most difficult ethical quandaries arising in medical research in developing countries and describes catastrophes in which sponsors of medical research have deliberately or inadvertently neglected ethical principles. Throughout, Macklin proposes many solutions to the problems of protecting research subjects, citing concrete examples to support her theories.

Although the title of this volume suggests a broader coverage, the emphasis is on ethical issues specific to human subjects in drug research trials. Specifically, Macklin gives particular (perhaps disproportionate) attention to trials in which the treatments evaluated are largely inapplicable to the population from which the research subjects are sampled. The exclusion of diagnostic, observational and epidemiologic research - modalities that frequently result in dramatic benefit to developing countries - may distort her conclusions.

Macklin sets out to establish the unacceptability of double standards in medical research in developing countries by examining four overlapping areas: disparities in the standard of care; the obligations of research sponsors to provide effective products; informed consent; and ethical reviews.

The author explores familiar ethical concepts such as distributive justice, vulnerability and exploitation to evaluate the problems in each of these areas. For example, are sponsors of medical research obligated to provide research subjects with diagnostic procedures and treatments that have been proven effective? In Macklin's opinion, sponsors, policy-makers, health officials and politicians in developing and industrialized countries should all bear this responsibility.

In her chapter on improving access to effective treatments after drug trials, Macklin presents a number of theoretical solutions to this central problem and substantiates these with concrete examples. She proposes theoretical and existing international collaborative partnerships, the relaxation of patents and differential pricing as some potential solutions. To her credit, Macklin does not assume that the good will of pharmaceutical companies will supersede the corporate profit motive. Instead, she emphasizes the promise of cooperative international efforts and public-private partnerships.

However, with regard to standards of care within research, she gives little attention to the importance of scientific design, a central but often overlooked part of protecting vulnerable participants. Although scientific criteria are an important part of the evaluation of research protocols, many ethical review committees do not include these in their evaluation. In addition, Macklin neglects to mention data safety monitoring boards, which periodically re- view outcome and safety data and play an important role in protecting study participants.

Although Macklin acknowledges that placing the burden of subjects' health care solely on research sponsors would likely impede medical progress and improvements in public health, she avoids taking a firm stance on whether access to proven-effective diagnotic manoeuvres and treatments must be included as part of a research proposal. The conflict between the imposition of such standards and the autonomy of local ethical committees is worth further discussion.

With regard to consent issues, Macklin does an admirable job of identifying the problems inherent in applying Western methodologies to resourcepoor and culturally diverse nations. She illustrates this with several examples of situations in which a Western approach has proven to be misguided. However, she proposes few alternatives and makes no mention of the paucity of empirical data on the subject. To date, little research has investigated how well subjects comprehend the information conveyed in "informed" consent forms. There are no studies on the best ways to obtain informed consent in diverse cultures or on subjects' comprehension of benefits and risks. Although in some areas there is a consensus that current methods to protect patients are ineffective, these processes have not come under scrutiny. For example, there have been no field studies in vulnerable populations to assess the applicability of informed consent forms. Obtaining empirical data is surely the crucial first step toward bridging the gap between bioethical theory and practice; the lack of such data is an important shortcoming to explore. 
Macklin explores the failures of ethical review committees in clinical research settings in developing countries. Despite a thorough review of the ways in which Western guidelines can fail patients, her evident support for the principles of the American model, in which a "more robust" system monitors the activities of institutional review boards (IRBs), hints dangerously at political imperialism. The proliferation of the procedures of such review boards can easily result in crippling overregulation. Collaborative studies conducted by federally funded institutions are a good example of this. A simple minimal risk village study (e.g., an examination of stool for the prevalence of parasites) conducted over a five-year period could require 40 separate IRB approvals (or renewals) - at great expense of money, time and effort. The fact that the multiplicity and redundancy of ethical reviews serves only to slow developments and thereby delay public health improvements is an issue that Macklin does not address.

Although we applaud Macklin's efforts to lobby on behalf of vulnerable populations of developing countries, we'd like to emphasize that ethical double standards are not confined to developing countries. There are 41 million Americans without health insurance and, according to Families USA (a national nonprofit, nonpartisan organization dedicated to the achievement of highquality affordable health care for all Americans), 65 million without prescription drug coverage. Although Macklin condemns the paternalistic paradigm often adopted by IRB committees in industrialized countries for medical research in developing countries, in her text she often supports the American medical system as a model. As in developing countries, subjects of drug trials conducted in the United States do not have access to all effective diagnostics and treatments once the trial is over. Access is limited by poverty in the United States as much as in any other country.

The grossly unequal provision of health care in the United States seems to escape Macklin's criticism. This blindside is something of a double stan- dard in itself. Given the lack of an ideal bioethical model in any Western nation, in any conflict between local IRBs and those of the sponsor's country the former should take precedence. This would better protect research subjects in vulnerable populations and also check the neocolonial approach adopted by many American IRBs.

Readers working in medical research in developing countries will inevitably find Macklin's frequent references to the following documents helpful: the Declaration of Helsinki (original and revised versions); the International Ethical Guidelines for Biomedical Research Involving Human Subjects, published by the Council for International Organizations for Medical Sciences; guidelines provided by the National Bioethics Advisory Commission; and UNAIDS documents. Macklin often quotes from these sources to highlight contradictions in the opinions by officials holding positions of influence within national and international agencies, such as the World Health Organization and the US Food and Drug Administration.

As we aspire to balance medical progress, public health and patient rights with the interests of industrialized countries, developing countries and pharmaceutical companies, we encounter a myriad of dilemmas. Whether one agrees or disagrees with Macklin's viewpoint, Double Standards in Medical Research in Developing Countries is a thoughtprovoking summation of the most troubling bioethical issues in clinical research trials and is, on balance, a worthy addition to the Law, Medicine and Ethics series of the Cambridge University Press.

\section{Kate Turner}

Asociación Benéfica PRISMA

Lima, Peru

Robert H. Gilman

Bloomberg School of Public Health

Johns Hopkins University

Baltimore, Md.

\section{Looking out a window at St. Michael's Hospital}

The cars outside scurry into parking holes:

an attendant administers tickets and takes money.

His gate goes up. Traffic breeds thick on the street.

Using paper bags for pillows, derelicts sleep in the square.

Townhouse roofs are worn from too much rain,

too much sun. An argument over money: Pay me then

and No, you stole it! A few joggers trot. Squads

of kids dispense their tortures. One pamphleteer

installs himself outside the hospital entrance

and is ignored. Sirens sound their Doppler calls;

pigeons swoop down on litter falling from pedestrians'

hands. A man wanders along the sidewalk; he looks lost.

This squat window is open, a perfect portal: no expanse, just a small demesne, a city as broad as its enclosure.

I turn around, a man lies with the sheets drawn

to his neck; only the damaged godhead is visible. A face

$\approx$ that loomed in life recedes each day, too gaunt

for arguments. I turn away. Outside, fewer cars pass.

The parking lot empties. Vagrants stir. Half a moon

presides over grief as patch of cityscape.

\section{Shane Neilson}

Family Physician

Guelph, Ont. 